\title{
Give Meaning to Alternative Methods to Animal Testing
}

doi:10.14573/altex.1907051

The $4^{\text {th }}$ advanced theoretical-training course "Give meaning to alternative methods to animal experimentation" was held in Genoa on November 29-30, 2018, hosted by the Italian Centro3R (Interuniversity Center for the Promotion of the 3Rs Principles in Teaching and Research) and LARF-DIMES (Laboratory for Analysis and Research in Physiopathology - Department of Experimental Medicine, Genova, Italy). The course, chaired by Prof. Arti Ahluwalia (CentroPiaggio, University of Pisa, Italy) and Prof. Anna Maria Bassi (LARF-DIMES), Director and Vice Director of Centro3R, respectively, with the valuable support of members of the Centro3R Operative Units of the Universities of Pisa and Genoa, and of the LARF Research Team, provided an update on new in vitro approaches.

The welcome addresses were given by Prof. Michela Tonetti, Director of DIMES, and Prof. Nicola Traverso (DIMES), who stressed the relevance of new in vitro approaches and technologies in toxicological research as well the role of universities in teaching and training future researchers.

Arti Ahluwalia (Centro3R, University of Pisa) introduced the course by stating that the role of universities is to provide a nurturing and liberal environment for teaching and research and therefore they represent an ideal place for the implementation and the promotion of the 3Rs. The Italian Interuniversity Center for the Promotion of the 3Rs Principles in Teaching and Research (Centro 3R) was recently established to promote rational and scientific thinking in experimental science through a multidisciplinary teaching and research approach, which includes all 3 Rs. Specifically, the Centro 3R will develop courses and credits on animal care and welfare and advanced in vitro methods, integrating them into bachelor's and master's curricula. In the research field, the Center is committed to the development of innovative 3Rs methods as well as knowledge and resource sharing.

Silvia Letasiova (MatTek In Vitro Life Science Laboratories, Bratislava, Slovakia) spoke on the development and validation of the EpiDerm in vitro skin irritation protocol for the evaluation of medical device extracts. EpiDerm can identify irritant polymer samples either in saline or in sesame oil device extracts. The use of the reconstructed tissue models as replacements for the rabbit intracutaneous test are being implemented into the ISO 10993 standards for the evaluation of medical device biocompatibility. Daniela Monti (Centro3R, University of Pisa) gave an overview on 3Rs approaches for predicting drug-induced eye irritation. The in vivo Draize eye test in rabbits is highly criticized for limited predictability, high costs, and ethical reasons. In vitro methods based on reconstituted corneal epithelium have been developed to assess eye irritation and are now also being evaluated for their ability to determine corneal permeability. Jan Markus (MatTek In Vitro Life Science Laboratories, Bratislava, Slovakia) explained the use of a reconstructed 3D human small intestine model (EpiIntestinal, MatTek Corporation) to predict drug toxicity and absorption/metabolism patterns. The EpiIntestinal model aims to improve the prediction of drug absorption and toxicity over models that are not organ- or species-specific, rely on cell lines or lack 3D architecture and functionality. It allows bidirectional drug penetration through the intestinal wall and expresses proteins involved in active drug transport and metabolism at physiological levels, which makes it ideal for modelling complex drug absorption profiles. Comparative studies revealed that the absorption of drug in EpiIntestinal mimics the in vivo profile more closely than the currently used Caco- 2 model. EpiIntestinal was also able to predict toxicity with much higher specificity and sensitivity than an animal model. Sonia Scarfî (Centro3R, University of Genova) reported on comparing silica-induced fibrosis in early metazoans and human cells.

Maria Grazia Cascone (Centro3R, University of Pisa) gave an overview on scaffolds used for tissue engineering, which serve as an artificial extracellular matrix (ECM) that hosts the cells and improves their survival, proliferation, and differentiation, enabling the formation of new tissue. In particular, protein/polysaccharide-based porous scaffolds for use as ECM substitutes in cardiac tissue engineering were discussed. These are based on blends of collagen or gelatin with alginate, produced by freeze-drying and ionic and chemical crosslinking. Tommaso Sbrana (IvTech s.r.1.) focused on 3-dimensional microfluidic in vitro models, which aim to reproduce the in vivo structure and environment of tissues to improve prediction of their response to stimuli or chemicals. This includes the exchange of nutrients and oxygen by means of microfluidics. Lauri Paasonen (UPM-Kymmene Oyj, Finland) covered the use of nanofibrillar cellulose (NFC) hydrogel (GrowDex) derived from wood as a matrix for 3D cell culture applications. NFC is biocompatible with human cells and tissues and allows free diffusion of small molecules, such as nutrients and oxygen. The 3D cell structures can be collected for detailed downstream analysis after cellulase treatment, which degrades GrowDex to soluble glucose with no observed adverse impact on cells. The shear-thinning property of GrowDex permits its use with automated dispensing systems at ambient temperature in high throughput screening-related 3D cell culture applications. Laura Pastorino (Centro3R, University of Genova) focused on the design of a $3 \mathrm{D}$ in vitro model of neuronal cultures to mimic the natural cell environment and enable physiological cell-cell interaction, gene expression, synaptogenesis and neurophysiological 
circuits to study the dynamics and connectivity of brain tissue. Chitosan microspheres were developed as a 3D scaffold to support the artificial neural network of primary neuronal cells (hippocampal and cortical neurons) and to carry out morphological and electrophysiological characterization. Chitosan is a promising scaffold for developing 3D neuronal networks for brain-on-achip microsystems.

Beatrice Ussia (Euroclone S.p,A., Milan, Italy) highlighted requirements of Good Cell Culture Practice, which include a detailed description of the cultured cells (origin, mode of culture initiation, morphology, differentiation state), authentication (karyotyping, DNA analysis, fingerprinting), and regular tests for cross-contamination. Furthermore, she explained the importance of defining standard operating procedures (SOPs), defining inter alia the composition of culture media, the nature of culture vessels and culture matrices, and the subculture procedure. Massimo Di Donato (TebuBio s.r.l., Milan, Italy) summarized the applications of human normal primary cells as in vitro models in plastic surgery, cosmetic research, drug metabolism, toxicity, immunology studies, and cancer research. Human primary cells representative of, e.g., pancreatic islets for diabetes studies, endothelial and muscle cells for regeneration studies, or vascular cells can be obtained from qualified companies. Moreover, iPSC-derived cells can be differentiated into several phenotypes, including cardiomyocytes and neuronal cells, which can be used as models for cardiotoxicity and neurodegenerative diseases, respectively.

In silico approaches for the prediction of chemical toxicity were the topic of the lecture of Prof. Paolo Milazzo (Centro3R, University of Pisa). He explained how a computational (in silico) model of a biological system is constructed, either by applying a machine learning method to a large amount of data about the system, or by following a mechanistic approach that consists of constructing a simulator based on the biophysical laws governing the system's dynamics. The approaches to computational modeling were described with a focus on their application in the analysis of chemical toxicity. When a deep knowledge of the biological system or enough data are available, computational approaches can lead to models that are more accurate than animal models.
Costanza Rovida (CAAT-Europe) covered the EU-ToxRisk project and its case studies on the read-across approach. Readacross exploits information on structurally related analogues to derive hypotheses about the activity of a new chemical and hence predict its toxicity without experimental testing. Large existing databases on traditional toxicological endpoints and mechanisms of action can be searched by data mining and cheminformatics tools. In addition, high-information-content techniques such as toxicogenomics and metabolomics can be utilized to generate and test these hypotheses. Anna Maria Bassi (Centro3R, University of Genova) spoke on replacing animal testing. Modern, human-specific models allow the understanding of human disease pathways at multiple biological levels, thus overcoming the drawbacks of animal models. This is a vibrant, multidisciplinary field which offers good future perspectives.

The course also offered training modules including working with the EpiDerm SIT-MD protocol for skin irritation testing of medical devices, the IVTech system technology as an in vitro model of connected cell cultures in dynamic conditions, a high throughput screening assay to evaluate GI toxicity of pharmaceuticals using EpiIntestinal, 2 and 3D cultures on Growdex, and the mini-microscope CytoSMART ${ }^{\mathrm{TM}}$ (Lonza.com) for real-time monitoring of cells in an incubator.

The evaluation questionnaires revealed satisfaction of all the participants, who requested the scheduling of other advanced courses. We thank Dr Helena Kandarova (MatTek), Dr Jane Spencer (UPM-Biofore), and all the specialists and researchers of Centro3R for their invaluable contribution to the success of the course.

\section{Anna Maria Bassi ${ }^{1,2}$ and Arti Ahluwalia ${ }^{1,3}$ \\ ${ }^{1}$ Centro 3R (Interuniversity Center for the Promotion of the 3Rs Principles in Teaching \& Research), Italy; ${ }^{2}$ LARF-DIMES, Department of Experimental Medicine (DIMES), University of Genoa, Genoa, Italy; ${ }^{3}$ Research Center E. Piaggio and Department of Information Engineering, University of Pisa, Pisa, Italy}

